\title{
Improving walking speed reduces hospitalization costs in outpatients with cardiovascular disease. An analysis based on a multistrata non-parametric test
}

Stefano Bonnini ${ }^{1 \dagger}$, Gianni Mazzoni ${ }^{2,3+}$, Michela Borghesi ${ }^{4}$, Giorgio Chiaranda ${ }^{5,6}$, Jonathan Myers ${ }^{7,8}$, Simona Mandini $2^{*^{*}}$, Andrea Raisi ${ }^{2}$, Sabrina Masotti ${ }^{2}$ and Giovanni Grazzi ${ }^{2,3}$

\begin{abstract}
Background: To assess the association between walking speed (WS) and its improvement on hospitalization rates and costs in outpatients with cardiovascular disease.

Methods: Six hundred forty-nine patients participating in an exercise-based secondary prevention program were studied. Patients were divided at baseline into two groups characterized by low and high WS based on the average WS maintained during a moderate $1-\mathrm{km}$ treadmill-walking test. WS and other covariates were grouped into three domains (demographic factors, medical history and risk factors), and used to estimate a propensity score, in order to create homogeneous groups of patients. All-cause hospitalization was assessed 3 years after baseline as a function of WS. Hospitalization and related costs were also assessed during the fourth-to-sixth years after enrollment. To test whether the hospitalization costs were related to changes in WS after 36 months, a multistrata permutation test was performed by combining within strata partial tests.

Results: The results support the hypothesis that hospitalization costs are significantly reduced in accordance with an improvement in WS. This effect is most evident among older patients, overweight or obese, smokers, and those without a history of coronary artery bypass surgery.

Conclusions: The present study supports growing evidence of an inverse association between WS, risk of hospitalization and consequent health-care costs. The joint use of propensity score and multistrata permutation approaches represent a flexible and robust testing method which avoids the possible effects of several confounding factors typical of these studies.
\end{abstract}

Keywords: Cardiovascular disease, Secondary prevention, Physical activity, Hospitalization costs, Permutation test

\footnotetext{
* Correspondence: simona.mandini@unife.it

'Stefano Bonnini and Gianni Mazzoni contributed equally to this work.

${ }^{2}$ Center for Exercise Science and Sport, University of Ferrara, Via Gramicia ,

35, 44121 Ferrara, Italy

Full list of author information is available at the end of the article
}

C C The Author(s). 2020 Open Access This article is licensed under a Creative Commons Attribution 4.0 International License, which permits use, sharing, adaptation, distribution and reproduction in any medium or format, as long as you give appropriate credit to the original author(s) and the source, provide a link to the Creative Commons licence, and indicate if changes were made. The images or other third party material in this article are included in the article's Creative Commons licence, unless indicated otherwise in a credit line to the material. If material is not included in the article's Creative Commons licence and your intended use is not permitted by statutory regulation or exceeds the permitted use, you will need to obtain permission directly from the copyright holder. To view a copy of this licence, visit http://creativecommons.org/licenses/by/4.0/ The Creative Commons Public Domain Dedication waiver (http://creativecommons.org/publicdomain/zero/1.0/) applies to the data made available in this article, unless otherwise stated in a credit line to the data. 


\section{Background}

Cardiovascular disease (CVD) is the leading cause of death globally, and is associated with an immense health and economic burden [1,2]. Prioritization of health behaviors (including physically active lifestyle), in addition to the treatment of established CVD, is a primary goal of numerous leading organizations worldwide to improve cardiovascular health and reduce healthcare costs $[2,3]$. Despite this overwhelming evidence, a considerable percentage of world's population has an insufficient level of physical activity (PA) [4-6], making it one of the most prevalent major CVD risk factors [7-9]. Recent estimates indicate that $\sim 5.3$ million deaths per year are directly attributable to insufficient PA, similar to smoking-related mortality globally $[7,10]$. In addition, inactivity imposes a large economic burden, recently quantified as $\sim \$ 120$ billion and $\sim € 80$ billion per year in the US and in the European Union, respectively [11, 12], mostly attributable to hospital costs [13]. Cost-effectiveness and feasibility of PA promotion in current clinical practice is well supported [7, 14-16]. This growing evidence and policy support has led to a number of alternative and pragmatic approaches to deliver proven and sustainable models that can be integrated into modern healthcare settings [7].

One key approach to increase PA and health across the population is to promote walking for recreation and transport [17-19]. Walking is the most common type of leisure-time PA among adults, and is simple, costeffective, and with a strong potential to improve public health [20, 21]. Walking speed (WS), measured as the time required to walk a given distance, is a common tool used to assess physical function [22]. WS has also been demonstrated to be inversely associated with disability, hospitalization, and survival in patients with CVD [23-26]. Indeed, WS has been suggested as the 'sixth vital sign' [27], and its clinical assessment has been recommended along with heart rate, breathing, temperature and pain [27]. WS determination requires low resources, time, and skills, making it suitable for routine assessment in clinical and research settings [22]. Slow walking speed (WS) has been associated with disability and higher risk for hospitalization and mortality in patients with CVD [23-26].

Evidence for the prognostic value of WS is largely based on a single measure at baseline. However, since PA habits can change during a given follow-up period, inferences based on a single measure at baseline could lead to erroneous conclusions [28]. The current study was conducted to investigate whether WS is associated with all-cause hospitalization costs, and whether serial changes in WS are accompanied by changes in longterm hospitalization costs. The joint application of propensity scoring and multistrata permutation represents a flexible and robust testing method, suitable in the presence of confounding factors typical of these studies.

\section{Methods}

\section{Study population}

Six hundred forty-nine consecutive patients were enrolled in an exercise-based secondary prevention program at the Center for Exercise and Sports Science at the University of Ferrara, Italy, and Department of Public Health, AUSL Ferrara, Italy. Patients were referred by their GP or cardiologist from at least 3 months up to several months after discharge. Patients were clinically stable based on the absence of need to modify overall therapy for at least 3 months before baseline examination, and with no symptoms at rest and during light to moderate exercise intensities during daily activities. The goal of the program was to promote long-term physically active lifestyles in outpatients with CVD. An unsupervised home program consisting of 30-60 min of brisk walking, at least 3-4 days per week, was recommended. Before admission, participants underwent a comprehensive clinical evaluation, including medical history. The study was approved by the Human Studies Committee of the University of Ferrara, no. 2213 , and all subjects gave written informed consent.

\section{Follow-up and hospitalization}

Patients were evaluated between October 1997 and January 2013. Follow-up sessions were created to motivate patients to promote and maintain physically active lifestyles. Followup booster visits were created to encourage patients and stimulate further involvement in physically active lifestyle. History of changes in PA or symptoms, and assessment of walking capacity were assessed at any visit. During these booster sessions, the subject's individualized goals (i.e. achievement of recommended level of PA) were discussed and were used as a basis for adjustment of the exercise prescription. Benefits associated with improved exercise capacity were explained, emphasizing the health consequences of being able to walk faster and longer.

On admission, quarterly during the first $2-3$ years, and thereafter twice per year, each patient performed a $1-\mathrm{km}$ treadmill-walking test at moderate perceived exercise intensity, at a value of $11-13 / 20$ on the $6-20$ Borg scale, as previously described (1 k-TWT) [29].

Benefits associated with improved WS were explained, emphasizing the health consequences of being able to walk faster and longer. Participants were flagged by the regional Health Service Registry of the Emilia-Romagna Region, which provided data on hospitalization. Any admission was considered an event. For patients experiencing $>1$ hospitalization, only the first event was considered in the analysis.

\section{Data analysis}

Several covariates were considered to account for possible confounding effects. Baseline WS reflects the general physical condition of subjects at a certain time [30], and 
may affect the potential of subjects to improve their WS. By using the median as a threshold value, patients were divided into two groups characterized by low and high WS at the baseline. The resulting dichotomous variable represented one covariate in the analysis. The other covariates were grouped into three domains: demographic factors, medical history and risk factors. At baseline, patient's CVD diagnosis was defined from the hospital discharge record. Blood chemistry analyses previously performed and left ventricular ejection fraction derived from a prior echocardiographic evaluation were registered. The demographic factors were age and gender variation 36 months after baseline. The medical history was represented by four dummy variables. Each variable indicates the possible presence of a specific event in a given patient's medical history. The events considered were myocardial infarction, coronary artery bypass graft (CABG), percutaneous transluminal coronary angioplasty (PTCA) and valvular repair/ replacement. The risk factors considered were glycaemia and five dummy variables indicating the presence of established risk factors (hypertension, angina, family history, hypercholesterolemia), or unhealthy behaviors (smoking status). To test whether the cost reduction increased as a function of the WS variation and vice-versa, the patients were classified according to whether the WS variation was high (greater than the median) or low (less than or equal to the median), and the two groups were compared in terms of mean cost variation.

Supplemental Table 1 shows the mean values and standard deviations of the hospitalization cost and of the covariates for each of the two groups.

\section{The model}

Let the random variable $C_{i t}$ represent the hospitalization cost of patient $i$ in the three-year period $t$, with $t=1,2$. The outcome $Y_{i}=C_{i 2}-C_{i 1}$ represents the cost variation for the $\mathrm{i}$-th patient and it is the response variable of the study. The sample of $n$ patients can be bipartite into two subgroups $H$ and $L$, depending on whether the increase in the WS walking speed after 36 months was high or low respectively. We tested the hypothesis of a significant negative effect of the WS increase on the cost variation. The problem can be represented as follows:

$$
Y_{i}=\mu_{L}+\delta_{H} w_{i}+\varepsilon_{i}
$$

where $\varepsilon_{i}$ is a zero-mean homoscedastic random error with $\operatorname{Var}\left(\varepsilon_{i}\right)=\sigma^{2}, \mu_{L}=E\left(Y_{i} \mid i \in L\right)$ is the mean cost variation for the group of patients with low WS increase, $w_{i}$ is the dummy variable that represents the membership to group $H$ and $\delta_{H}$ is the effect of the WS increase on the cost variation.

The testing problem.

The hypotheses of the problem are:

$$
H_{0}: \delta_{H}=0
$$

and

$$
H_{1}: \delta_{H}<0 .
$$

In the null hypothesis $H_{0}$, there is no effect of the WS increase because $\mu_{H}=E\left(Y_{i} \mid i \in H\right)=\mu_{L}+\delta_{H}=\mu_{L}$, and in the alternative hypothesis $H_{1}$ there is a negative effect thus $\mu_{H}<\mu_{L}$.

To take into account the possible confounding effects of covariates, we classified the patients into homogeneous strata according to the confounding factors, performed two-sample within-stratum tests and combined them.

\section{The nonparametric method}

A methodological solution can be found in the family of combined permutation tests [31-34], suitable for several complex testing problems $[35,36]$. The hypotheses (2) and (3) can be broken down into $\mathrm{k}>1$ partial null hypotheses and $\mathrm{k}>1$ partial alternative hypotheses. The $\mathrm{k}$ partial tests are combined to solve the global test.

The $p$-values of the partial and global tests are computed according to the null permutation distribution of the test statistics (under the assumption of exchangeability in the null hypothesis). Without loss of generality, let us assume that the $\mathrm{j}$-th partial null hypothesis is rejected for large values of the test statistic $T_{j}$ and $L_{j}(x)=\operatorname{Pr}\left[T_{j} \geq\right.$ $x]$ be the significance level function. Hence, by denoting with $T_{j}^{(b)}$ the value of the $\mathrm{j}$-the partial test statistic corresponding to the $\mathrm{b}$-th permutation of the dataset, with $b=1, \ldots, B$, and with $T_{j}^{(0)}$ the observed value of the test statistic, the $p$-value of the $j$-th partial test is

$$
l_{j}=L_{j}\left(T_{j}^{(0)}\right)=\sum_{b \geq 1} I\left(T_{j}^{(b)} \geq T_{j}^{(0)}\right) / B
$$

where $B$ is the total number of permutations and $I(x)$ is the indicator function that takes value 1 when $x$ is true. For computational convenience, a random sample of the set of permutations (draws with replacement) can be used and estimated $p$-values can be computed instead of the exact ones, $\hat{l}_{j}=\left[\sum_{b \geq 1} I\left(T_{j}^{(b)} \geq T_{j}^{(0)}\right)+0.5\right] /(B+1)$. The value of the combined test statistic corresponding to the b-th permutation is $T_{c o m b}^{(b)}=\psi\left[L_{1}\left(T_{1}^{(b)}\right), \ldots\right.$, $\left.L_{k}\left(T_{k}^{(b)}\right)\right]$ and the estimated $p$-value of the global test is $\hat{l}_{c o m b}=\hat{L}\left(T_{\text {comb }}^{(0)}\right)$. The combination function $\psi$ must be non increasing in the arguments and must attain its supremum (strictly greater than the significance level $\alpha$ ) when at least one argument tends to zero.

In case of significance of the global test, it may be of interest to know which partial tests determine the global significance. However, a suitable control of the familywise error rate (probability of wrong rejection of one 
partial null hypothesis) must be applied, to avoid that the probability of type I error (wrong rejection of the global $H_{0}$ ) may exceed the significance level $\alpha$. Thus the significance of the $j$-th partial test must be assessed by comparing its $p$-value with an adjusted (reduced) significance level or, equivalently, the adjusted partial $p$ value ${ }_{a d g} \hat{l}_{j}$ (greater than or equal to the original partial $p$-value $\hat{l}_{j}$ ) with the significance level $\alpha$ [37]. A powerful solution is the so called Bonferroni-Holm procedure [38], which is preferable to the conservative Bonferroni rule where ${ }_{\text {adg }} \hat{l}_{j}=k \hat{l}_{j}$. According to the BonferroniHolm procedure

$$
{ }_{\operatorname{adg}} \hat{l}_{(j)}=\max _{r \leq j}\left\{\min \left[1,(k-r+1) \hat{l}_{(j)}\right]\right\}
$$

where $\hat{l}_{(j)}$ denotes the $\mathrm{j}$-th ordered estimated partial $p$-value. Given that we are considering a multiple test with the goal of determining contribution of the partial tests to the possible global significance by computing the Bonferroni-Holm adjustment of the $p$-values, the Tippett function seems to be the suitable choice for the tests combination.

\section{The propensity score approach for the stratification}

One of the main confounding factors is the WS at the baseline and, since we are interested to assess its possible confounding effect, it is considered separately with respect to the other confounders. All the other factors are too many to be considered separately for the stratification purpose. Thus the propensity score technique is applied [39, 40]. Let us assume that the membership to $\mathrm{H}$ of patient $i$ follows a Bernoulli distribution with parameter $\pi_{i}$ and that the relationship between $\pi_{i}$ and the confounders can be represented by the logit model $\log \left[\pi_{i} /\left(1-\pi_{i}\right)\right]=\beta_{0}+\sum_{j} \beta_{j} x_{i j}+\xi_{i}$, where $x_{i j}$ is the observed value of the $j$-th confounding factor on patient $i, \xi_{i}$ is the random error of the model and $\beta_{0}, \cdots, \beta_{k}$ are unknown parameters. The estimated propensity score is

$$
\hat{\pi}_{i}=\exp \left(\hat{\beta}_{0}+\sum_{j} \hat{\beta}_{j} x_{i j}\right) /\left[1+\exp \left(\hat{\beta}_{0}+\sum_{j} \hat{\beta}_{j} x_{i j}\right)\right]
$$

Where $\hat{\beta}_{0}, \cdots, \hat{\beta}_{k}$ are suitable estimates of $\beta_{0}, \cdots, \beta_{k}$ respectively.

To define homogeneous strata of patients, according to the confounding factors, the estimated propensity score quantiles can be used as thresholds to determine the membership to strata. For example, the tertiles can be used to split data into three groups that represent homogeneous strata of patients.

\section{Multistrata test}

Patients were initially divided into two groups based on WS performance during the $1 \mathrm{k}$-TWT at baseline, and classified as fast walkers or slow walkers for WS values above and below the median value, respectively. Next, patients were classified into low and high improvers according to variation in WS 3 years after baseline. To assess the association between WS, WS variation and incidence of 3 year hospitalization, we constructed Kaplan-Meier curves. Significantly correlated variables were entered into a fully adjusted Cox regression model, with first hospitalization as the outcome.

By jointly considering (i) the bipartition of the patients according to the median of the WS at the baseline (1: low, 2:high) and (ii) the tripartition of the patients according to the tertiles of the estimated propensity score based on the other confounding factors (1:low, 2: medium, 3:high), six different strata are determined and then the test on the effect of the WS increase on the cost variation can be broken down into six partial tests.

The null hypothesis of the problem can be represented as follows

$$
H_{0}: \cap_{\nu} \cap_{s} H_{0, \nu s}
$$

where $v=1,2$ denotes the group according to criterion (i) and $s=1,2,3$ the group according to criterion (ii), and $H_{0}, v s$ is the partial null hypothesis of no effect within stratum $(v, s) . H_{0}$ is true if and only if all the partial null hypotheses are true. Similarly, the alternative hypothesis can be formally written as

$$
H_{1}: \cup_{v} \cup_{s} H_{1, \nu s}
$$

Where $H_{1, \text { vs }}$ is the partial alternative hypothesis of significant (negative) effect within stratum $(v, s) . H_{1}$ is true when at least one partial alternative hypothesis is true.

For the random errors $\varepsilon_{1}, \cdots, \varepsilon_{n}$ defined in (1), the usual independence assumption typical of the parametric tests is not necessary. Since a permutation approach is applied, the weaker condition of exchangeability is assumed.

In order to perform the propensity score analysis, all the covariates of direct interest (age, sex, BMI, AMI, CABG, PTCA, Valve repair/replacement, hypertension, total cholesterol, creatinine levels, fasting glucose, smoking habit, and medications) and the information about the belonging of each patient to one of the two groups based on the walking speed variation, were included in a Minitab software worksheet. By means of a binary logistic regression, $P$-values of every covariate were investigated in order to determine their significance, whose level was set at $P \leq 0.1$. Regression equation coefficients were also estimated in order to evaluate the effect of each covariate on the probability that they are linked to WS variation. As said, baseline WS was considered separately. We then tested the hypothesis that hospitalization costs, within each stratum and overall, were lower for the individuals classified as High improvers compared to 
those classified as Low improvers running the multistrata permutation test with the software NPC Test. The significance level was set at the 0.05 . Those who were never admitted to hospital were considered as cost zero $(€=0)$.

\section{Results}

Baseline walking speed and 3-year hospitalization

Baseline characteristics of the participants are presented in Table 1. The median WS at baseline was $4.2 \mathrm{~km} / \mathrm{h}$. During the 3 years after baseline, 227 subjects $(35 \%$ of the sample) were hospitalized with an incidence of 43 and $27 \%$ for slow and fast walkers, respectively. The cumulative risk of hospitalization by groups of WS is presented in Fig. 1 (log rank, $P<0.0001$ ). In the logistic binary regression, only age, BMI variation, history of CABG and smoking status affected the probability of being in the fast walkers group. In the presence of history of CABG, the probability was higher, while the influence of age, BMI increase and being a current smoker were not significant. Cox analysis showed that, compared to the slowest group, the HR for hospitalization was lower in the fastest group (HR 0.85, 95\%CI 0.73-0.99, $P=0.038$ ). After adjustments for confounders, every $1 \mathrm{~km} /$ hour increase in WS was associated with a $21 \%$ reduction in risk of hospitalization (HR 0.79, 95\% CI 0.71-0.89, $P<0.001$ ).

\section{Walking speed improvement and 4 to 6 years' hospitalization}

WS among the entire study population improved 3 years after baseline from $4.2 \pm 1.0$ to $5.0 \pm 1.1 \mathrm{~km} /$ hour. The

Table 1 Characteristics of the subjects at the baseline by groups of walking speed

\begin{tabular}{|c|c|c|c|}
\hline & $\begin{array}{l}\text { All subjects } \\
(n=649\end{array}$ & $\begin{array}{l}\text { SLOW walkers } \\
(n=332)\end{array}$ & $\begin{array}{l}\text { FAST walkers } \\
(n=317)\end{array}$ \\
\hline Walking speed (km/h) & $4,2(1,0)$ & $3,4(0,7)$ & $5,0(0,6)$ \\
\hline \multicolumn{4}{|l|}{ General } \\
\hline Age (yr) & $63(9)$ & $66(9)$ & $60(9)$ \\
\hline Gender (M/F) & $568 / 81$ & $263 / 69$ & $305 / 12$ \\
\hline $\mathrm{BMI}\left(\mathrm{kg} / \mathrm{m}^{2}\right)$ & $27,9(3,8)$ & $28,4(4,0)$ & $27,4(3,5)$ \\
\hline LV ejection fraction (\%) & $56(10)$ & $55(10)$ & $58(9)$ \\
\hline \multicolumn{4}{|l|}{ Risk factor } \\
\hline Current smoking (\%) & 5 & 3 & 6 \\
\hline Hypertension (\%) & 60 & 70 & 55 \\
\hline Famili history (\%) & 53 & 44 & 62 \\
\hline Fasting glucose (mg/dL) & $106(25)$ & $108(26)$ & $104(23)$ \\
\hline Total cholesterol (mg/dL) & $194(41)$ & $196(41)$ & $191(42)$ \\
\hline HDL cholesterol (mg/dL) & $50(12)$ & $50(12)$ & $50(12)$ \\
\hline Serum truglycerides (mg (dL) & $135(67)$ & $137(37)$ & $132(68)$ \\
\hline Serum creatinine (mg/dL) & $1,09(0,3)$ & $1,13(0,3)$ & $1,05(0,2)$ \\
\hline \multicolumn{4}{|l|}{ Medical history } \\
\hline CABG (\%) & 56 & 60 & 52 \\
\hline Myocardial infarction (\%) & 21 & 18 & 27 \\
\hline PTCA (\%) & 8 & 6 & 9 \\
\hline Valvular repair/replacement (\%) & 11 & 13 & 8 \\
\hline Other (\%) & 4 & 3 & 4 \\
\hline \multicolumn{4}{|l|}{ Medications } \\
\hline ACE inhibitor or ARB (\%) & 55 & 60 & 50 \\
\hline Aspirin (\%) & 75 & 73 & 77 \\
\hline$\beta$-blockers (\%) & 59 & 56 & 62 \\
\hline Calcium antagonists (\%) & 13 & 14 & 12 \\
\hline Diuretics (\%) & 15 & 24 & 5 \\
\hline Statins (\%) & 55 & 49 & 61 \\
\hline
\end{tabular}

Values are presented as mean (standard deviation, SD) or \%. Abbreviations: ACE Angiotensin-Converting Enzyme, ARB Angiotensin Receptor Blocker, BMI Body Mass Index, CABG Coronary Artery Bypass Graft, HDL High-density lipoproteins, LV Left Ventricular, PTCA Percutaneous Transluminal Coronary Angioplasty, stenting or both 


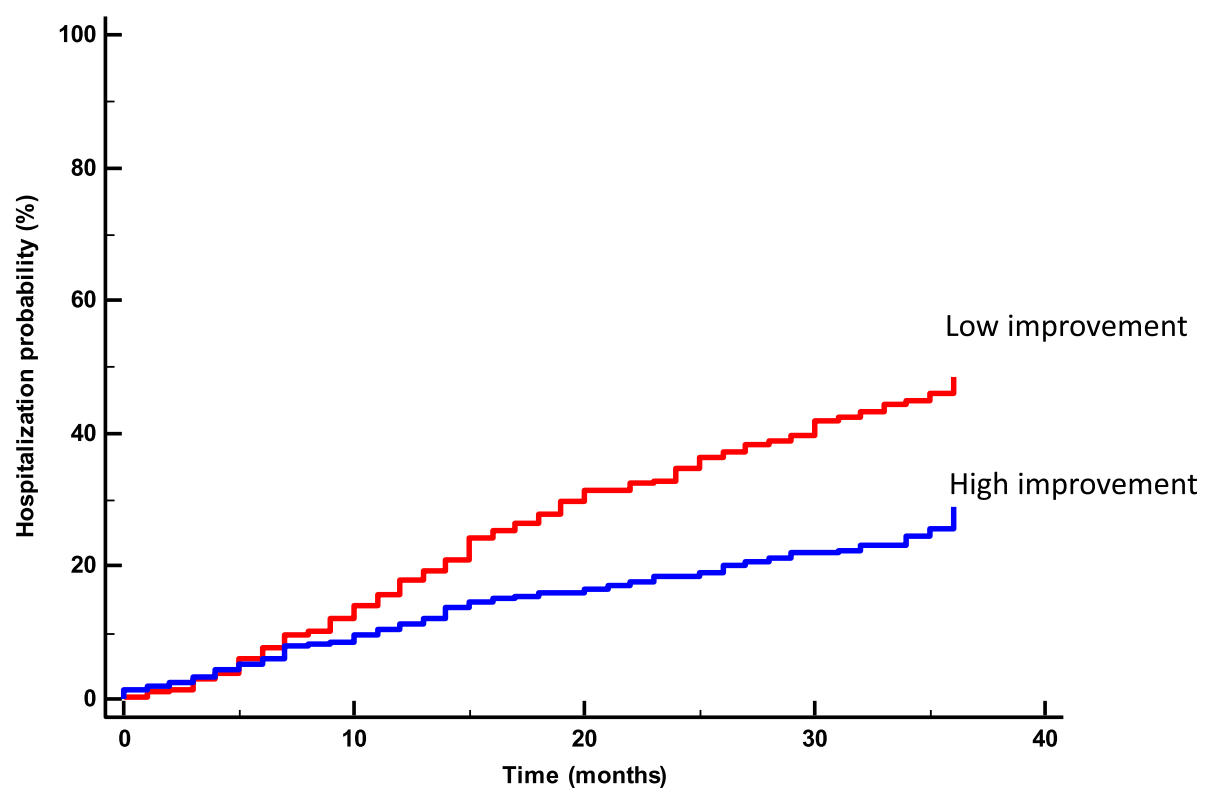

Fig. 1 Rate of hospitalization during 36 months after enrolment stratified according to walking speed at baseline

improvements in WS in the slow and fast groups at baseline were $3.4 \pm 0.7$ to $4.3 \pm 1.0 \mathrm{~km} /$ hour, and $5.0 \pm 0.6$ to $5.7 \pm 0.7 \mathrm{Km} /$ hour, respectively. During 4 -to- 6 years after baseline, 211 subjects (36\% of the sample) were hospitalized. Figure 2 shows the Kaplan-Meier survival curves for hospitalization by groups of WS improvement. The incidences of hospitalization were 44 and
$28 \%$ for Low and High improvers (log rank $P<0.0001$ ). Among the interactions between WS change and covariates, only age, gender, BMI variation, family history of CVD, and history of CABG were statistically significant. The lower hospitalization rate for WS improvement persisted after adjustment for confounders. Cox regression analysis showed that, compared to the slowest group,

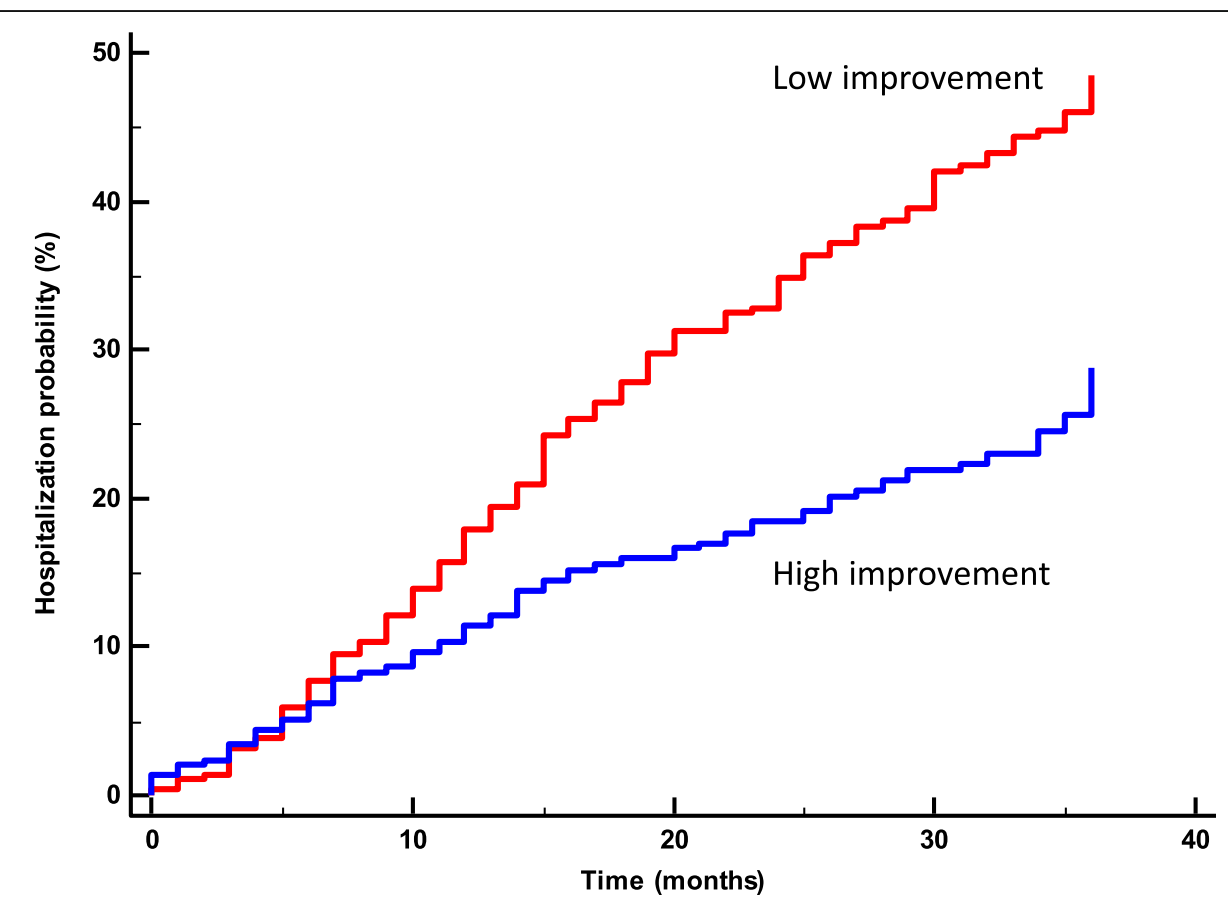

Fig. 2 Rate of hospitalization 36 to 72 months after enrolment stratified according to walking speed improvement 
the HR for hospitalization was lower in the fastest group (HR 0.51, 95\%CI $0.38-0.68, P=0.0001$ ). Every $1 \mathrm{~km} /$ hour increase in WS was associated with an adjusted $39 \%$ reduction in risk for hospitalization (HR 0.61, 95\%CI 0.49-0.76, $P<0.0001$ ).

The logistic binary regression, to study the relationship between the propensity score and covariates related to demographic information, medical history and risk factors, was performed with a stepwise method to determine the significance of the coefficients. At the significance level $\alpha=0.05$, only age, BMI variation, CABG and smoking affect the probability to be in group $\mathrm{H}$ (high increase of WS). In the presence of CABG, the mentioned probability is higher; instead, the effect on the mentioned probability caused by age, BMI increase and being a smoker is negative. The application of (5) allows to estimate the propensity score of each patient as function of age, BMI variation, CABG and smoking (Table 2).

The first tertile of the estimated propensity scores is 0.44 , the second tertile is 0.55 . The median of the WS at the baseline resulted $4.2 \mathrm{~km} / \mathrm{h}$. In Table 3 we can see that, according to the combined multi-strata permutation test, the hypothesis of no effect must be rejected at $\alpha=0.05$ in favor of the hypothesis that an increase of WS lowers the hospitalization costs (global $P$-value = 0.020).

According to the adjusted $P$-values, the global significance must be attributed to the sub-groups "low WS at the baseline and low propensity score" (stratum 1) and "high WS at the baseline and low propensity score" (stratum 4).

As reported in Table 4, stratum 1 consists of patients with a mean age equal to 70 (oldest group among the three with low WS at baseline), a mean BMI increase of 1.37 (highest difference among the three groups with low WS at the baseline), a 5\% of smokers, and 38\% with CABG (maximum and minimum respectively, when the WS at the baseline is low). Among the patients with high WS at baseline, stratum 4 is the oldest (mean age 64), the one with highest BMI increase (1.20 mean variation), with the lowest percentage of patients with CABG (29\%) and the highest percentage of smokers (19\%). Thus, the effect of WS increase on the cost reduction is significant in old patients with great BMI increase. The absence of CABG facilitates the significant effect of the WS increase on the costs, which is relevant among the smokers. Hospitalization costs in the first, second and third propensity score tertile per patient were reduced from 1131 to $301 €$, from 798 to $338 €$, and from 1057 to $295 €$ among low and high improvers, respectively.

\section{Discussion}

In this cohort of outpatients with CVD referred to an exercise-based secondary prevention program, slower WS at baseline was associated with a higher risk of allcause hospitalization over 1 to 3 years. These results further support the inverse relationship between walking capacity and long-term risk of morbidity and mortality

Table 2 Propensity score analysis: binary logistic regression for dummy high speed increase (dependent variable)

\begin{tabular}{lll}
\hline Explanatory variable & Estimated coefficient & $\boldsymbol{p}$-value \\
\hline Constant & 22,053 & 0,000 \\
Demographic covariates & & 0,000 \\
Age & $-0,0383$ & 0,0 \\
Gender (1:Male; 0:Female) & n.s. & 0,000 \\
Body Mass Index Variation & $-0,2465$ & \\
Medical history & & 0,78 \\
Myocardial infarction & n.s. & 0,003 \\
Coronary artery bypass & 0,4988 & \\
Percutaneous transluminal coronary angioplasty & n.s. & 1,65 \\
Valvular replacement & n.s. & \\
Risk factors & & 0,048 \\
Glycemia & n.s. & \\
Smoke & $-0,7870$ & 0,46 \\
Hypertension & n.s. & \\
Angina & n.s. & \\
Familiar history & n.s. & \\
Hypercholesterolemia & n.s. & \\
\hline
\end{tabular}

n.s. Non significant

$\mathrm{a}=0.05$ 
Table 3 Multistrata permutation test on the effect of walking speed increase on hospitalization cost

\begin{tabular}{lllll}
\hline $\begin{array}{l}\text { Average walking } \\
\text { speed at baseline }\end{array}$ & Propensity score & Sample size & $P$-value & Adjusted $\boldsymbol{P}$-value \\
\hline Low & Low & 124 & 0,003 & 0,020 \\
& Medium & 110 & 0,640 & 0,955 \\
& High & 98 & 0,673 & 0,955 \\
High & Low & 90 & 0,005 & 0,023 \\
& Medium & 115 & 0,785 & 0,955 \\
& High & 112 & 0,283 & 0,738 \\
\hline
\end{tabular}

in outpatients with ischemic heart disease [41, 42], and chronic heart failure [43], as well as in those undergoing cardiac surgery [24]. The second notable finding was the reduced rates and costs of hospitalization associated with the improvement in WS between 4 and 6 years after baseline. After propensity score analysis and through permutation testing we observed a significant cost reduction for the high improvers compared to the low improvers. In particular, the significance of the hospitalization cost reduction as a consequence of an increase in WS can be attributed to the oldest patients, those with high BMI, the absence of coronary artery bypass surgery, and smokers. We have empirical evidence that this is true for both the WS levels at the baseline.

These findings are in agreement with previous data demonstrating that similar PA interventions may account for between $€ 1120$ and $€ 15,860$ per quality-adjusted lifeyear gained, which can be more cost-effective than drugbased interventions $[7,43]$. These results further support growing evidence related to reduced health care costs in more active individuals $[13,44]$. The findings of this study extend to a broad spectrum of individuals with a wide range of age (28-88 years), WS (1.2-7.1 km/h), and demographic and clinical characteristics.

Several factors likely contribute to explain these results. Physical activity has an important role in the prevention and management of $>40$ chronic conditions.7 However, while established CVD risk factors are assessed routinely in clinical practice $[45,46], \mathrm{PA}$ is commonly not assessed [47]. In this study we assessed WS, which reflects the combined functioning of the cardiorespiratory, nervous and musculoskeletal systems $[48,49]$. The moderate WS determined by the endurance treadmill-walking protocol employed in this study was inversely associated with survival in a large cohort of outpatients with CVD [50]. Favorable associations between WS improvement and hospitalization rate and costs resulted independent from age and clinical history. In addition, we jointly utilized two different but complementary statistical methods - combined permutation and propensity score - to investigate differences in hospitalization rates and costs.

Another relevant aspect of this study concerns the utilization of serial measurements over a long follow-up. The prognostic value of WS in ageing and chronic diseases is well documented. However, this evidence is mostly based on a unique assessment at baseline. Since lifestyles can change over a given follow-up period, there is a great deal of value in assessing WS serially [28].

\section{Implications for policy}

This study further supports the use of exercise-based secondary prevention programs in a broad range of patients with CVD [47]. Traditional interventions in patients with CVD consist primarily of supervised exercise sessions delivered in a hospital [51]. However, notable evidence indicates that these models are often neither financially viable nor sustainable $[52,53]$. Thus, creating and implementing alternative approaches have been advocated [5]. Home-based models have been shown to be effective and safe [54-56], and are less time consuming and logistically easier for many patients $[5,57,58]$. These models, similar to the one presented herein, are

Table 4 Strata characterization in terms of rilevant confounding factors

\begin{tabular}{llllll}
\hline $\begin{array}{l}\text { Average walking } \\
\text { speed at baseline }\end{array}$ & Propensity score & Mean age & Mean BMI var. & Coronary art. bypass (\%) & Smoke (\%) \\
\hline Low & Low & 69,66 & 1,37 & 37,90 & 4,84 \\
& Medium & 66,96 & 0,03 & 72,73 & 2,73 \\
& High & 59,01 & $-1,02$ & 72,45 & 2,04 \\
High & Low & 64,41 & 1,20 & 28,89 & 18,89 \\
& Medium & 61,35 & 0,43 & 53,91 & 1,74 \\
& High & 54,03 & $-0,27$ & 67,86 & 0,89 \\
\hline
\end{tabular}


an option for those patients who cannot easily access hospital- or facility-based programs [58].

\section{Strengths of the study}

First, there was a large sample size of patients across a wide range of age and functional capacity. Second, the walking test protocol is simple, and a potential easy tool to apply in clinical practice. Furthermore, walking is the most frequently performed form of physical activity $[59,60]$, and the preferred activity by insufficiently active individuals, regardless of age and sex [61]. Yet, the incorporation of goals meaningful to a given patient for performing activities of daily living such moderate WS has been recommended [53]. Fourth, the non-restrictive inclusion criteria employed is likely to reflect real-world clinical practice. Fifth, the study was based on an analysis of administrative data and used an analytic matching approach and a multistrata test to control for potential confounding. This permitted us to assess specific characteristics of the patient subgroups for which the effect of walking speed variation was significant, increasing the validity of findings. Finally, to our knowledge, no other study has examined the effects of improvement in WS on hospitalization costs in cardiac outpatients during a follow-up of this length.

\section{Limitations of the study}

First, our results may not apply to different settings, which may have different policies for health services use and costs. Second, this approach does not fully adjust for the severity of cardiovascular risk profile or comorbidity [62-64], including injury-related hospitalization. Third, this study was carried out in patients who chose to participate in an exercise-based secondary prevention program and thus, they may not be representative of the general population with similar cardiovascular conditions. Fourth, given the modest number of women, the findings of this study should be used with caution among females. Fifth, adherence to the recommended home program was not determined, and thus, a causal relationship between the "dose" of PA and the WS improvement is not possible. However, we recently demonstrated in a randomized controlled trial in older outpatients with CVD that the intervention produced significant increase in habitual PA, associated with improved health outcomes [65-67].

\section{Conclusions}

The present study contributes to growing evidence that supports the inverse association between slow WS and risk of hospitalization, and lower health-care costs. These results provide evidence that moderate WS is a simple, potentially useful, and clinically important indicator of health for outpatients with CVD. The test may help health professionals identify less fit patients which may require greater use of health services, ultimately requiring greater monetary expenditures. Finally, the findings may help health professionals seeking a simple method to reduce the prevalence of sedentary behavior that remains extremely high among patients with CVD.

\section{Supplementary Information}

The online version contains supplementary material available at https://doi. org/10.1186/s12913-020-05874-3.

Additional file 1: Table S1. Hospitalization cost variation and covariates (mean \pm SD) versus walking speed variation (dichotomized).

\begin{abstract}
Abbreviations
BMI: Body Mass Index; WS: Walking speed; CVD: Cardiovascular disease; PA: Physical activity; 1 k-TWT: 1-km treadmill-walking test; CABG: Coronary artery bypass graft; PTCA: Percutaneous transluminal coronary angioplasty stenting or both; ACE: Angiotensin-Converting Enzyme; ARB: Angiotensin Receptor Blocker; HDL: High-density lipoproteins; LV: Left Ventricular
\end{abstract}

\section{Acknowledgements}

The authors acknowledge Dr. Cinzia Nanini and Dr. Stefano Palazzi of the Public Health Department of AUSL Ferrara (Italy) for facilitating the exercise testing sessions. We would also wish to thank Prof. Emidia Vagnoni for contributing to the determination of hospitalization costs and with some considerations on the subject.

\section{Authors' contributions}

$\mathrm{SB}, \mathrm{MB}$ GM and GG have contributed to the conception and design of the work; $\mathrm{SB}$ and $\mathrm{MB}$ analysed and interpreted the patient data regarding the hospitalization costs; GC, JM, SM, AR, SM and GM have drafted the work and revised it; all authors read and approved the final manuscript.

\section{Funding}

Not applicable.

\section{Availability of data and materials}

The dataset generated and analysed during the current study are not publicly available due to limits determined by the rules of our health system in terms of sensitive data management. However, data are available from the corresponding author on reasonable request.

\section{Ethics approval and consent to participate}

The study was approved by the Human Studies Committee of the University of Ferrara, no. 22-13. The written consent was obtained from all the participants prior to taking part in this study.

\section{Consent for publication \\ $\mathrm{N} / \mathrm{A}$}

\section{Competing interests}

The authors declare that they have no competing interests.

\section{Author details}

${ }^{1}$ Department of Economics and Management, University of Ferrara, Ferrara, Italy. ${ }^{2}$ Center for Exercise Science and Sport, University of Ferrara, Via Gramicia , 35, 44121 Ferrara, Italy. ${ }^{3}$ Public Health Department, AUSL Ferrara, Ferrara, Italy. ${ }^{4}$ Center for Modelling Computing and Statistics, University of Ferrara, Ferrara, Italy. ${ }^{5}$ Public Health Department, AUSL Piacenza, Piacenza, Italy. ${ }^{6}$ General Directorship for Public Health and Integration Policy, Emilia-Romagna Region, Bologna, Italy. 'Division of Cardiology, VA Palo Alto, Palo Alto, CA, USA. ${ }^{8}$ Stanford University School of Medicine, Stanford, CA, USA. 
Received: 25 May 2020 Accepted: 28 October 2020

Published online: 17 November 2020

\section{References}

1. Mendis S, Puska P, Norrving B. Global Atlas on cardiovascular disease prevention and control, Geneva, Switzerland, http://whqlibdoc.who.int/ publications/2011/9789241564373_eng.pdf 2011.

2. Benjamin EJ, Muntner P, Alonso A, On behalf of the American Heart Association Council on Epidemiology and Prevention Statistics Committee and Stroke Statistics Subcommittee, et al. Heart disease and stroke statistics-2019 update. A report from the American Heart Association. Circulation. 2019;139. https://doi.org/10.1161/CIR.0000000000000659.

3. Knuuti J, Wijns W, Saraste A, et al. ESC guidelines for the diagnosis and management of chronic coronary syndromes the task force for the diagnosis and management of chronic coronary syndromes of the European Society of Cardiology. Eur Heart J. 2019;00:1-71. https://doi.org/ 10.1093/eurheartj/ehz425.

4. Fletcher GF, Ades PA, Kligfield P, American Heart Association Exercise, Cardiac Rehabilitation, and Prevention Committee of the Council on Clinical Cardiology, Council on Nutrition, Physical Activity and Metabolism, Council on Cardiovascular and Stroke Nursing, and Council on Epidemiology and Prevention, et al. Exercise standards for testing and training: a scientific statement from the American Heart Association. Circulation. 2013;128:873-934.

5. Lavie CJ, Arena R, Swift DL, et al. Exercise and the cardiovascular system: clinical science and cardiovascular outcomes. Circ Res. 2015;117:207-19.

6. Katzmarzyk PT, Lee IM, Martin CK, Blair SN. Epidemiology of physical activity and exercise training in the United States. Prog Cardiovasc Dis. 2017:60:3-10

7. Lobelo F, Rohm Young D, Sallis R, et al. Routine assessment and promotion of physical activity in healthcare settings. A scientific statement from the American Heart Association. Circulation. 2018;137. https://doi.org/10.1161/ CIR.0000000000000559.

8. Centers for Disease Control and Prevention. State Indicator Report on Physical Activity. Atlanta: US Department of Health and Human Services; 2014. 11/Lobelo 2018.

9. Mozaffarian D, Benjamin EJ, Go AS, on behalf of the American Heart Association Statistics Committee; Stroke Statistics Subcommittee, et al. Executive summary: heart disease and stroke statistics - 2016 update: a report from the American Heart Association. Circulation. 2016;133:447-54.

10. Wen $C P, W u X$. Stressing harms of physical inactivity to promote exercise. Lancet. 2012;380:192-3.

11. Carlson SA, Fulton JE, Pratt M, Yang Z, Adams EK. Inadequate physical activity and health care expenditures in the United States. Prog Cardiovasc Dis. 2015;57:315-23.

12. ISCA/Cebr, June 2015. The economic cost of physical inactivity in Europe. In: An ISCA/Cebr Report, (http://inactivity-time-bomb.nowwemove.com/ download-report/The\%20Economic\%20Costs\%20of\%20Physical\%2 Olnactivity\%20in\%20Europe. Accessed 12 July 2020.

13. Mozaffarian D, Benjamin EJ, Go AS, on behalf of the American Heart Association Statistics Committee and Stroke Statistics Subcommittee, et al. Heart disease and stroke statistics-2015 update. A report from the American Heart Association. Circulation. 2015;131:e29-e322.

14. Global Action Plan for the Prevention and Control of Noncommunicable Diseases 2013-2020. Geneva, Switzerland: World Health Organization; 2018 https://www.who.int/ncds/prevention/physical-activity/global-action-plan-2 018-2030/en/. Accessed 28 June 2020.

15. Eakin EG, Brown WJ, Marshall AL, Mummery K, Larsen E. Physical activity promotion in primary care: bridging the gap between research and practice. Am J Prev Med. 2004;27:297-303.

16. Grandes G, Sanchez A, Sanchez-Pinilla RO, et al. Effectiveness of physical activity advice and prescription by physicians in routine primary care: a cluster randomized trial. Arch Intern Med. 2009:169:694-701.

17. Oja P, Kelly P, Murtagh EM, et al. Effects of frequency, intensity, duration and volume of walking interventions on CVD risk factors: a systematic review and meta-regression analysis of randomised controlled trials among inactive healthy adults. Br J Sports Med. 2018;52:769-75.

18. Reis RS, Salvo D, Ogilvie D, et al. Scaling up physical activity interventions worldwide: stepping up to larger and smarter approaches to get people moving. Lancet. 2016:388:1337-48.

19. Hardman AE, Morris JN. Walking to health. Br J Sports Med. 1998;32:184.
20. Francis K, Williamson T, Kelly $P$, et al. Continuous walking and time- and intensity matched interval walking: Cardiometabolic demand and postexercise enjoyment in insufficiently active, healthy adults. J Sports Sci. 2020. https://doi.org/10.1080/02640414.2020.1803717.

21. Kelly P, Murphy M, Mutrie N. The health benefits of walking. In: Mulley C, Gebel K, Ding D, editors. Walking. 2017; Transport and Sustainability, vol. 9: Emerald Publishing. p. 61-79. https://doi.org/10.1108/S2044994120170000009004

22. Kamiya K, Hamazaki N, Matsue Y, et al. Gait speed has comparable prognostic capability to six-minute walk distance in older patients with cardiovascular disease. Eur J Prev Cardiol. 2018;25(2):212-9.

23. Chaudhry SI, McAvay G, Chen S, et al. Risk factors for hospital admission among older persons with newly diagnosed heart failure: findings from the cardiovascular health study. J Am Coll Cardiol. 2013;61:635-42.

24. Afilalo J, Eisenberg MJ, Morin JF, et al. Gait speed as an incremental predictor of mortality and major morbidity in elderly patients undergoing cardiac surgery. J Am Coll Cardiol. 2010;56:1668-76.

25. Rolland $Y$, Lauwers-Cances $V$, Cesari $M$, et al. Physical performance measures as predictors of mortality in a cohort of community-dwelling older French women. Eur J Epidemiol. 2006;21:113-22.

26. Centers for Disease Control and Prevention. CDC Vital Signs. More People Walk to Better Health. 1600 Clifton road NE, Atlanta, GA 30333. http://www. cdc.gov/vitalsigns/pdf/2012-08-vitalsigns.pdf. Accessed 28 June 2020.

27. Fritz S, Lusardi M. Walking speed: the sixth vital sign. J Geriatr Phys Ther. 2009;32:46-9.

28. Steffen-Batey L, Nichaman MZ, Goff DC Jr, et al. Change in level of physical activity and risk of all-cause mortality or reinfarction: the Corpus Christi heart project. Circulation. 2000;102:2204-9.

29. Chiaranda G, Myers J, Mazzoni G, et al. Peak oxygen uptake prediction from a moderate, perceptually regulated, $1-\mathrm{km}$ treadmill walk in male cardiac patients. J Cardiopulm Rehabil Prev. 2012;32:262-9.

30. Granacher U, Völler H. Gait speed is not magic, but is prognostically important in older patients. Eur J Prev Cardiol. 2018;25(2):209-11. https://doi. org/10.1177/2047487317744053. Epub 2017 Nov 22. PMID: 29164921.

31. Pesarin F. Multivariate permutation tests with applications in biostatistics. Chichester: Wiley; 2001.

32. Pesarin F, Salmaso L. Permutation tests for complex data. Theory, applications and software. Chichester: Wiley; 2010.

33. Arboretti Giancristofaro R, Bonnini S. Nonparametric directional tests in the presence of confounding factors and categorical data. Stat Appl. 2009;VII(1): 87-103.

34. Bonnini S, Corain L, Marozzi M, Salmaso L. Nonparametric hypothesis testing. Rank and Permutation Tests with Applications in R. Chichester: Wiley; 2014

35. Bonnini S, Prodi N, Salmaso L, Visentin C. Permutation approaches for stochastic ordering. Commun Stat-Theor M. 2014;43(10-12):2227-35.

36. Arboretti Giancristofaro R, Bonnini S, Salmaso L. Employment status and education/employment relationship of PhD graduates from the University of Ferrara. J App Stat. 2009;36(12):1329-44.

37. Arboretti Giancristofaro R, Bolzan M, Bonnini S, Corain L, Solmi F. Advantages of closed testing method in multiple comparison procedure. Commun Stat-Simul C. 2012;41:746-63.

38. Holm S. A simple sequentially rejective multiple test procedure. Scand J Stat. 1979;6:65-70

39. Rosenbaum PR, Rubin DB. Assessing sensitivity to an unobserved binary covariate in an observational study with binary outcome. J R Stat Soc B. 1983:45(2):212-8.

40. Rosenbaum PR, Donald BR. "The Central Role of the Propensity Score in Observational Studies for Causal Effects." Biometrika. 1983;70(1):41-55. JSTOR, www.jstor.org/stable/2335942. Accessed 12 July 2020.

41. Beatty AL, Schiller NB, Whooley MA. Six-minute walk test as a prognostic tool in stable coronary heart disease. Arch Int Med. 2012;172:1096-102.

42. Matsuzawa Y, Konishi M, Akiyama E, et al. Association between gait speed as a measure of frailty and risk of cardiovascular events after myocardial infarction. J Am Coll Cardiol. 2013;61:1964-72.

43. Garrett S, Elley CR, Rose SB, O'Dea D, Lawton BA, Dowell AC. Are physical activity interventions in primary care and the community cost-effective? A systematic review of the evidence. Br J Gen Pract. 2011;61:e125-33.

44. Peeters GMEE, Mishra GD, Dobson AJ, Brown WJ. Health care costs associated with prolonged sitting and inactivity. Am J Med. 2014:46(3): $265-72$. 
45. Strath SJ, Kaminsky LA, Ainsworth BE, on behalf of the American Heart Association Physical Activity Committee of the Council on Lifestyle and Cardiometabolic Health and Cardiovascular, Exercise, Cardiac Rehabilitation and Prevention Committee of the Council on Clinical Cardiology, and Council, et al. Guide to the assessment of physical activity: clinical and research applications: a scientific statement from the American Heart Association. Circulation. 2013;128:2259-79.

46. Barnes, P. and C. Schoenborn. "Trends in adults receiving a recommendation for exercise or other physical activity from a physician or other health professional." NCHS Data Brief. 2012;86:1-8 .

47. Forman DE, Arena $\mathrm{R}$, Boxer $\mathrm{R}$, et al. Prioritizing functional capacity as a principal end point for therapies oriented to older adults with cardiovascular disease a scientific statement for healthcare professionals from the American Heart Association. Circulation. 2017; 135:e894-918.

48. Myers J, Prakash M, Froelicher $V$, et al. Exercise capacity and mortality among men referred for exercise testing. N Engl J Med. 2002;346: 793-801.

49. Simonsick EM, Fan E, Fleg JL. Estimating cardiorespiratory fitness in wellfunctioning older adults: treadmill validation of the long distance corridor walk. J Am Geriatr Soc. 2006;54:127-32.

50. Chiaranda G, Bernardi E, Codecà L, et al. Treadmill walking speed and survival prediction in men with cardiovascular disease: A 10-year follow-up study. BMJ Open. 2013;3:e003446.

51. Moghei M, Turk-Adawi K, Isaranuwatchai W, et al. Cardiac rehabilitation costs. Int J Cardiol. 2017;244:322-8.

52. Sandesara PB, Lambert CT, Gordon NF, et al. Cardiac rehabilitation and risk reduction: time to "rebrand and reinvigorate". J Am Coll Cardiol. 2015;65: 389-95.

53. Arena $\mathrm{R}$. The case for cardiac rehabilitation post-PCl and why we aren't meeting our goal. American College of Cardiology. 2015; Available at: http://www.acc.org/latest-incardiology/articles/2015/08/13/14/10/thecasefor-cardiac-rehabilitation-post-pci. Accessed 22 Oct 2015.

54. Cowie A, Thow MK, Granat MH, Mitchell SL. A comparison of home and hospital-based exercise training in heart failure: immediate and long-term effects upon physical activity level. Eur J Cardiovasc Prev Rehabil. 2011;18: 158-66

55. Kraal JJ, Peek N, Van den Akker-Van Marle ME, Kemps HM. Effects of homebased training with telemonitoring guidance in low to moderate risk patients entering cardiac rehabilitation: short-term results of the FIT@home study. Eur J Prev Cardiol. 2014;21(suppl):26-31.

56. Ramadi A, Haennel RG, Stone JA, Arena R, Threlfall TG, Hitt E, Aggarwal SG Haykowsky M, Martin BJ. The sustainability of exercise capacity changes in home versus center-based cardiac rehabilitation. J Cardiopulm Rehabil Prev. 2015;35:21-8.

57. Schopfer DW, Forman DE. Cardiac rehabilitation in older adults. Can J Cardiol. 2016;32(9):1088-96

58. Dalal HM, Zawada A, Jolly K, Moxham T, Taylor RS. Home based versus Centre based cardiac rehabilitation: Cochrane systematic review and metaanalysis. BMJ. 2010;340:b5631.

59. Belza B, Walwick J, Shiu-Thornton S, Schwartz S, Taylor M, LoGerfo J. Older adult perspectives on physical activity and exercise: voices from multiple cultures. Prev Chronic Dis. 2004;1(4):A09 Epub 2004 Sep 15.

60. Burton E, Lewin G, Boldy D. Physical activity preferences of older home care clients. Int J Older People Nursing. 2015:10(3):170-8.

61. Booth ML, Bauman A, Owen N, Gore CJ. Physical activity preferences, preferred sources of assistance, and perceived barriers to increased activity among physically inactive Australians. Prev Med. 1997;26(1):131-7.

62. Baber $U$, Boffetta P. Improving fitness to achieve health: shifting the focus from theory to practice. J Am Coll Cardiol. 2015;65:2101-3.

63. Rosano C, Brach J, Studenski S, et al. Gait variability is associated with subclinical brain vascular abnormalities in high-functioning older adults. Neuroepidemiology. 2007;29:193-200.

64. Heald CL, Fowkes FG, Murray GD, et al. Risk of mortality and cardiovascular disease associated with the ankle-brachial index: systematic review. Atherosclerosis. 2006:189:61-9.

65. Tonet E, Maietti E, Chiaranda G, et al. Physical activity intervention for elderly patients with reduced physical performance after acute coronary syndrome (HULK study): rationale and design of a randomized clinical trial. BMC Cardiovasc Disord. 2018;18:98
66. Campo G, Tonet E, Chiaranda G, et al. Exercise intervention to improve functional capacity in older adults after acute coronary syndrome. J Am Coll Cardiol. 2019 Dec 10;74(23):2948-50.

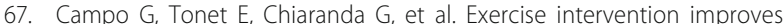
quality of life in older adults after myocardial infarction: randomised clinical trial. Heart. 2020;Epub ahead of print. https://doi.org/10.1136/ heartjnl-2019-316349.

\section{Publisher's Note}

Springer Nature remains neutral with regard to jurisdictional claims in published maps and institutional affiliations.
Ready to submit your research? Choose BMC and benefit from:

- fast, convenient online submission

- thorough peer review by experienced researchers in your field

- rapid publication on acceptance

- support for research data, including large and complex data types

- gold Open Access which fosters wider collaboration and increased citations

- maximum visibility for your research: over $100 \mathrm{M}$ website views per year

At BMC, research is always in progress.

Learn more biomedcentral.com/submissions 